\title{
Altered expression of the Arabidopsis ortholog of DCL affects normal plant development
}

\section{Journal Article}

Author(s):

Bellaoui, Mohammed; Gruissem, Wilhelm

Publication date:

2004-09

Permanent link:

https://doi.org/10.3929/ethz-b-000161149

Rights / license:

In Copyright - Non-Commercial Use Permitted

Originally published in:

Planta 219(5), https://doi.org/10.1007/s00425-004-1295-5 


\section{Altered expression of the Arabidopsis ortholog of $D C L$ affects normal plant development}

\begin{abstract}
The DCL (defective chloroplasts and leaves) gene of tomato (Lycopersicon esculentum Mill.) is required for chloroplast development, palisade cell morphogenesis, and embryogenesis. Previous work suggested that DCL protein is involved in $4.5 \mathrm{~S}$ rRNA processing. The Arabidopsis thaliana (L.) Heynh. genome contains five sequences encoding for DCL-related proteins. In this paper, we investigate the function of AtDCL protein, which shows the highest amino acid sequence similarity with tomato DCL. AtDCL mRNA was expressed in all tissues examined and a fusion between AtDCL and green fluorescent protein (GFP) was sufficient to target GFP to plastids in vivo, consistent with the localization of AtDCL to chloroplasts. In an effort to clarify the function of $A t D C L$, transgenic plants with altered expression of this gene were constructed. Deregulation of AtDCL gene expression caused multiple phenotypes such as chlorosis, sterile flowers and abnormal cotyledon development, suggesting that this gene is required in different organs. The processing of the $4.5 \mathrm{~S}$ rRNA was significantly altered in these transgenic plants, indicating that AtDCL is involved in plastid rRNA maturation. These results suggest that AtDCL is the Arabidopsis ortholog of tomato DCL, and indicate that plastid function is required for normal plant development.
\end{abstract}

M. Bellaoui $(\bowtie) \cdot$ W. Gruissem

Institute of Plant Sciences,

Swiss Federal Institute of Technology,

8092 Zurich, Switzerland

E-mail: mohammed.bellaoui@utoronto.ca

Tel.: 1-416-9465774

Fax: 1-416-9788548

Present address: M. Bellaoui

Department of Biochemistry,

University of Toronto, Medical Sciences Building,

Room 5326, 1 King's College Circle, Toronto,

Ontario, M5S 1A8, Canada
Keywords Arabidopsis. Defective chloroplasts and leaves Chloroplast development $\cdot$ Plastid ribosomal RNA

Abbreviations DCL: Defective chloroplasts and leaves - GFP: Green fluorescent protein

\section{Introduction}

As in the eubacteria, the plastid rRNA genes are arranged in an operon and are transcribed as a large precursor RNA, in the order $16 S, 23 S$ and $5 S$ (Kössel et al. 1985). However, plastid rRNA operons have some features that are distinct from those of prokaryotic rRNA. For example, in Chlamydomonas reinhardii the region corresponding to the $5^{\prime}$ end of the eubacterial $23 S$ gene is divided into $7 S$ and $3 S$ rRNAs (Rochaix and Darlix 1982). Sequence comparisons have revealed that the $3 S$ and $7 S$ rRNAs are not unique to plastids, but are equivalent to the $5^{\prime}$ terminal regions of the eubacterial $23 S$ rRNA (Rochaix and Darlix 1982).

In the chloroplasts of flowering plants the $7 \mathrm{~S}$ and $3 \mathrm{~S}$ rRNAs are absent, but the $3^{\prime}$ terminus of the $23 S$ gene is split off as a separate gene termed $4.5 \mathrm{~S}$ rRNA (Edwards et al. 1981). This small rRNA is separated from the mature $23 S$ gene by short spacer that is removed from the precursor rRNA posttranscriptionally (Kössel et al. 1982). The primary transcript containing the $16 S, 23 S$, $4.5 S$ and $5 S$ rRNAs is processed into the $16 S$ and the $5 S$ rRNAs, and the $23 S-4.5 S$ rRNA precursor. The latter is subsequently cleaved into the $23 \mathrm{~S}$ and $4.5 \mathrm{~S}$ rRNAs (Kössel et al. 1982, 1985; Strittmatter and Kössel 1984).

While there is extensive information about the organization of plastid rRNA operons (reviewed by Harris et al. 1994), little is known about the enzymatic mechanisms of rRNA maturation. Mutants blocked at various stages of rRNA cleavage would be helpful to identify nuclear genes that play critical roles in plastid rRNA processing. A gene of tomato, defective chloroplasts and 
leaves $(D C L)$, which is required for rRNA maturation has been identified in our laboratory. $D C L$ is ubiquitously expressed and encodes a small protein that is required for chloroplast development, palisade cell morphogenesis, and embryogenesis (Keddie et al. 1996; Bellaoui et al. 2003). Examination of the expression of several nuclear and plastid genes has suggested that DCL protein is involved in the processing of $4.5 \mathrm{~S}$ rRNA (Bellaoui et al. 2003).

The objective of the present work was to gain insight into DCL function in Arabidopsis thaliana, a particularly good model system for this study because of the many genetic resources available. We have characterized the AtDCL gene, one of the Arabidopsis homologs of $D C L$. We show that the AtDCL gene is expressed ubiquitously and that AtDCL protein is efficiently imported into chloroplasts in vivo, confirming that AtDCL is plastid-localized. We also demonstrate that several phenotypic changes are induced when the expression of $A t D C L$ is deregulated in transgenic Arabidopsis plants. Furthermore, we show that $4.5 S$ rRNA maturation is affected in these transgenic plants, implying that $A t D C L$ is most likely the Arabidopsis ortholog of DCL.

\section{Materials and methods}

Plant material and growth conditions

Seeds of Arabidopsis thaliana (L.) Heynh. were sown in optima soil (http://www.optima.magnet.ch) and kept for 2 days at $4^{\circ} \mathrm{C}$ before being moved into the growth chamber. Plants were grown at $22^{\circ} \mathrm{C}$ under long-day growth conditions ( $16 \mathrm{~h}$ light $/ 8 \mathrm{~h}$ dark). Transgenic Arabidopsis seeds were surface-sterilized, and plated on Murashige and Skoog medium containing hygromycin $\left(35 \mu \mathrm{g} \mathrm{ml}^{-1}\right)$. Hygromycin-resistant seedlings were transferred to soil and grown in long-day conditions in the growth chamber.

$3^{\prime}$ and $5^{\prime}$ rapid amplification of cDNA ends

( $3^{\prime}$ and $5^{\prime}$ RACE)

Total RNA was extracted from Arabidopsis leaves. First-strand cDNA from total RNA was prepared using the Advantage RT-for-PCR Kit (Clontech). 3' and 5' RACE experiments were performed using the Marathon cDNA Amplification kit (Clontech) and the following $A t D C L$-specific primers:

- For 5' RACE:

- 5'-CTC TTA AAA TCC GGG TGA TGC CCT ACC-3'

- 5'-GCG AAA ATG TCT GAG GAT GAA ACT GTC-3'

- 5'-TGG CTG AGG TCT CAC CCC AAA ACC-3'.
- For 3' RACE:

- 5'-GAT GCT ACT TCC TTA TCA TCC TGA ATG-3'

- 5'-GAC AGT TTC ATC CTC AGA CAT TTT CGC-3'

- 5'-CTC GGT TCC ACT ACA AGG TTT TGG GGT-3'.

Construction of the AtDCL::GFP fusion

and the transformation vectors

The open reading frame of the AtDCL gene was PCRamplified using primers that contained an extra restriction site for $N c o$ I. The amplified fragment was digested with $N c o$ I and cloned in-frame into the $N c o$ I site of the green fluorescent protein (GFP) expression vector pGFP-MRC (Rodriguez-Concepcion et al. 1999) to express the fusion protein AtDCL::GFP. The nucleotide sequence of the resulting construct (named pAtDCL::GFP) was checked by sequencing.

For overexpression of AtDCL::GFP in A. thaliana, the pAtDCL::GFP construct was digested with HindIII to isolate the 35S::TL::AtDCL::GFP::Ter fragment. This fragment was then ligated into the pGPTVHPT plant vector. The generated construct was introduced into Agrobacterium tumefaciens cells for plant transformation. Plants were inoculated with A. tumefaciens by dipping aerial parts of the plants for $20 \mathrm{~s}$ in dipping solution as described by Clough and Bent (1998).

To generate the antisense AtDCL construct, the open reading frame of the AtDCL gene was PCRamplified by using primers that contained extra restriction sites. The amplified fragment was digested and cloned in antisense orientation into HindIII-XbaI sites of the plant vector pGPTV-HPT. The generated construct was further sequenced for confirmation and introduced into A. tumefaciens cells for plant transformation.

\section{Subcellular localization}

Protoplasts were isolated as described by Spangenberg and Potrykus (1995).Confocal imaging was performed using a Leica confocal laser-scanning microscope.

\section{RNA gel blot analysis}

RNA was extracted as described by Bonhomme et al. (1991). Northern blot experiments were conducted as described by Ausubel et al. (1990). $10 \mu \mathrm{g}$ of RNA was hybridized to random-primed AtDCL probe. The probe was generated by PCR amplification of the coding region of $A t D C L$. 

predicted amino acid sequences of tomato (Lycopersicon esculentum) DCL and genes. The alignment was created using MultAlin and Boxshade (ExPASy Molecular Biology Server and Boxshade server). Gaps that were introduced to improve the alignment are represented by dashes. Dark-shaded boxes indicate amino acids that are identical in at least three sequences and lighter shaded boxes indicate conserved amino acids. Stars indicate the DCLdomain
Fig. 1 Alignment of the Arabidopsis thaliana-related

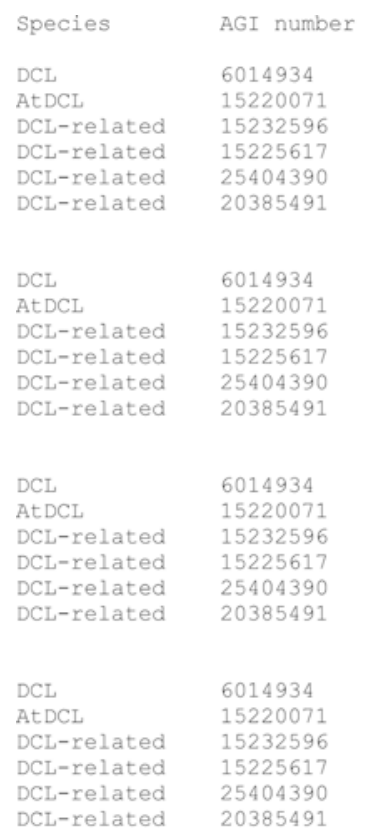

Protein gel blot analysis

Total Protein extracts were prepared as described by Barkan (1998) and resuspended in Laemmli buffer (Laemmli 1970). Protein electrophoresis and western blotting were conducted as described by Grelon et al. (1994). Immunoblots were developed using the Super Signal kit (Pierce Chemical Co.).

\section{Results}

Arabidopsis homologous of DCL

Sequence comparison of tomato DCL with proteins in current databases revealed significant similarity to five sequences from the Arabidopsis genome-sequencing

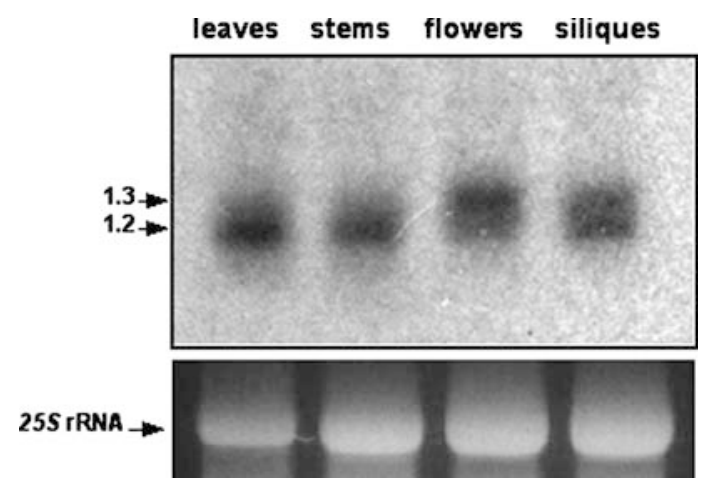

Fig. 2 RNA-blot analysis of the AtDCL gene. A 10- $\mu$ g sample of total RNA from each organ was loaded in each lane. The blot was hybridized with AtDCL cDNA probe. The numbers on the left indicate the approximate length of each transcript in kilobases. After ethidium bromide coloration of the gel, the cytosolic $25 \mathrm{~S}$ rRNA is shown in the lower panel as a loading control
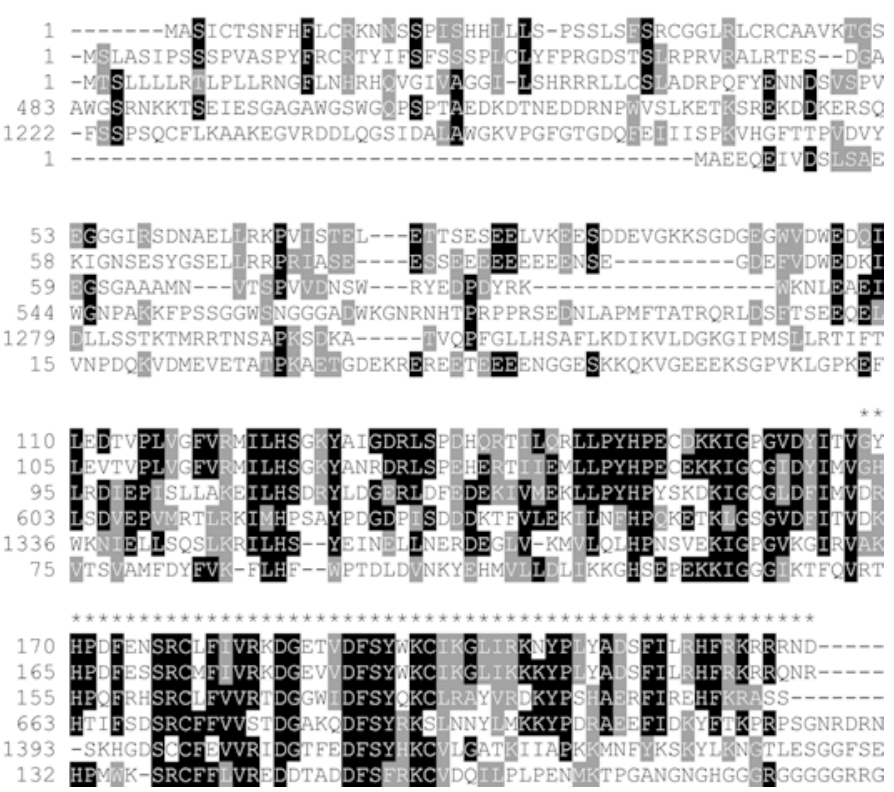

project (Fig. 1). These sequences showed no significant homology to any protein sequence in the database other than DCL, except for a $D C L$-related gene, which is much larger than tomato DCL (AGI 2504390) and encodes a protein related to RNA polymerase subunit. One of these $D C L$-related sequences exhibited the highest amino acid sequence similarity with tomato DCL and was designated AtDCL. It shows 68\% similarity and $60 \%$ identity over the entire open reading frame (Fig. 1). The highest sequence identity was seen at the C-terminus (92\% similarity and $83 \%$ identity), which contains the DCL-domain found in all DCL-related proteins (Bellaoui et al. 2003). Analysis of these Arabidopsis DCL-related proteins using common targeting prediction programs (http://www.inra.fr/Internet/ Produits/Predotar/) suggested that only AtDCL is localized in the chloroplast. We suggest, therefore, that AtDCL is the Arabidopsis ortholog of DCL.

\section{Expression pattern of $A t D C L$}

To begin the study of the function of $A t D C L$, expression analysis of the AtDCL gene was undertaken. A screen of the EST database identified two expressed sequence tags
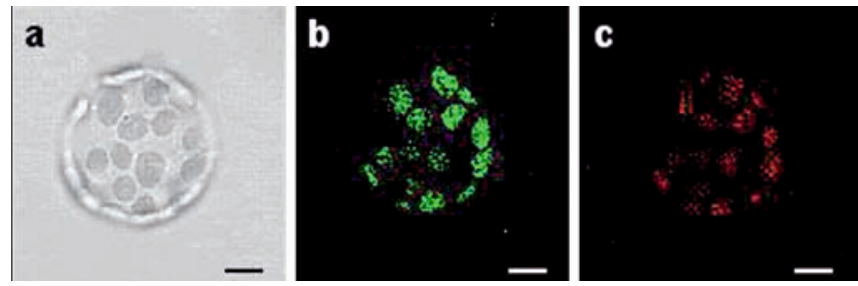

Fig. 3a-c The AtDCL protein imports the GFP into chloroplasts in vivo. Bright-field (a), fluorescence (b) and chlorophyll autofluorescence (c) images of a protoplast from a stably transformed Arabidopsis plant with AtDCL::GFP fusion. Bars $=10 \mu \mathrm{m}$ 
Fig. 4a-c Phenotype of A. thaliana plants harboring the $35 S: \because$ AtDCL: $: G F P$ transgene. a Four-week-old wild-type (top) and AtDCL::GFP-2 transgenic (bottom) plants. b Two-monthold wild-type (left) and AtDCL::GFP-2 mutant (right) plants. c Variation in cotyledon number and shape in the progeny of the AtDCL::GFP-2 line
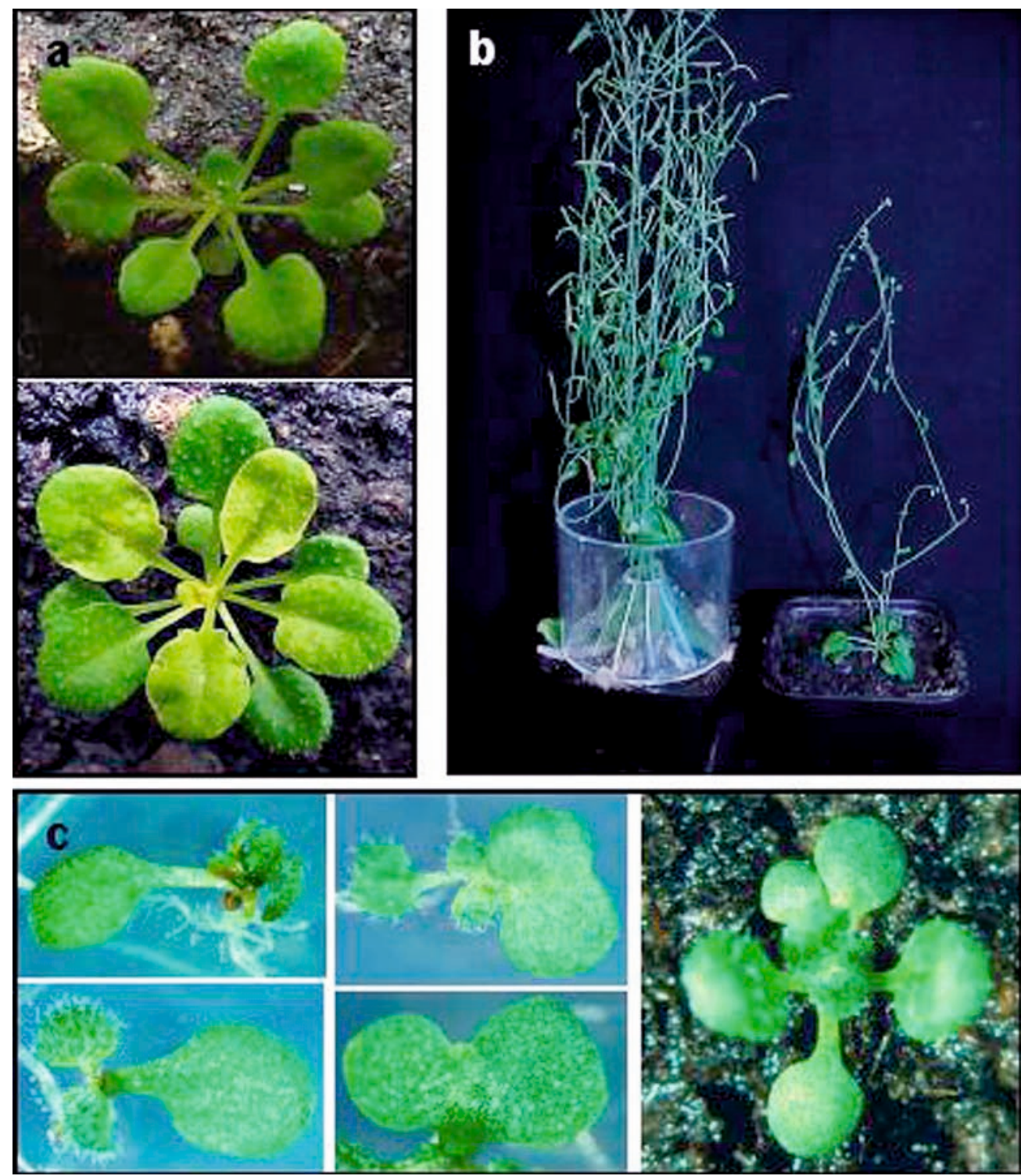

(accession numbers: AI994762 and N38199). Neither of these cDNA sequences is complete (391 and $417 \mathrm{bp}$ ), and so $5^{\prime}$ and $3^{\prime}$ RACE experiments were performed using total RNA from leaves. The sequence of the fulllength cDNA of AtDCL was then determined (1,151 bp).

Using AtDCL cDNA as probe, the expression pattern of AtDCL was examined by northern blot experiments. The AtDCL gene was expressed in all organs examined, including leaves, stems, flowers, and siliques (Fig. 2). This is consistent with the expression pattern of tomato $D C L$, which is also expressed in all types of tissues and stages of development examined (Keddie et al. 1996). A $1.2-\mathrm{kb}$ transcript was detected in the four organs examined, consistent with the size determined by sequencing the full-length cDNA of AtDCL. However, a second transcript of $1.3 \mathrm{~kb}$ was detected in flowers, and siliques. Since AtDCL genes are quite different from each other at the level of DNA sequence, we assume that this $1.3-\mathrm{kb}$ transcript in flowers and siliques corresponds to differentially spliced transcript or resulted from an alternative transcript termination.

\section{AtDCL targets GFP to the chloroplast in vivo}

To confirm that AtDCL is targeted to plastids in vivo, an in-frame fusion between the coding regions of AtDCL and the gene for GFP was made and used for stable transformation of Arabidopsis. The expression of AtDCL::GFP fusion in protoplasts isolated from a stably transformed Arabidopsis plant was examined by confocal laser-scanning microscopy. The results of these experiments clearly show that the fusion protein colocalized with the chloroplast chlorophyll (Fig. 3). Without the putative transit peptide, GFP signals were dispersed in the cytoplasm and nucleus (data not shown). Thus, AtDCL is able to target proteins to plastids in vivo, confirming that AtDCL is a plastidlocalized protein. 


\section{Analysis of AtDCL::GFP fusion overexpression}

To explore the function of the AtDCL gene, phenotypic and molecular analyses of transgenic Arabidopsis lines overexpressing the AtDCL::GFP fusion were performed. For this, the AtDCL::GFP fusion was cloned downstream of the CaMV 35S promoter. This promoter was chosen because it is known to be constitutively expressed in Arabidopsis, an expression pattern that matches that of the AtDCL gene. This construct was transformed into Arabidopsis wild-type plants (ecotype Columbia).

In total, 15 transformants were obtained after selection on Murashige and Skoog medium containing hygromycin. When transferred to soil, $12 \mathrm{~T} 1$ transformants showed similar developmental defects and were further analyzed. The transformants were pale green (Fig. 4a), had chlorotic and poorly fertile flowers and their growth was extremely retarded compared to the wild type (Fig. 4b). The results presented here are all derived from studies of one of these transgenic lines, named AtDCL::GFP-2. Segregation analysis of the T2 generation indicated that a single T-DNA was inserted into the AtDCL::GFP-2 line. Northern blot analysis revealed that this line accumulated a very high level of AtDCL::GFP transcript and GFP fluorescence indicated that the AtDCL::GFP fusion protein accumulated to high levels and was correctly localized in the plastids (Fig. 3 and data not shown). Therefore, these results suggest that the developmental defects observed in transgenic lines resulted from an overexpression of AtDCL::GFP fusion protein.

When germinated in vitro, the $\mathrm{T} 2$ progeny of the AtDCL::GFP-2 line contained seedlings with abnormal cotyledon development (seedlings with one, three, fused or unequal cotyledons) (Fig. 4c). This phenotype is consistent with the role of tomato $D C L$ during embryogenesis (Bellaoui et al. 2003).

To test whether AtDCL protein is involved in $4.5 \mathrm{~S}$ rRNA processing, we examined the expression of the small rRNA in the transgenic plants. As expected, the expression pattern of $4.5 S$ rRNA was severely affected in the AtDCL::GFP-2 mutant (Fig. 5). In contrast, 5S rRNA accumulated normally (Fig. 5). Together, these data suggest that overexpression of AtDCL::GFP affects $4.5 S$ rRNA formation, which in turn affects normal plastid function and plant development. Therefore, we suggest that overexpression of AtDCL::GFP behaves as a dominant negative mutation and indicates that $A t D C L$ might play the same function as tomato $D C L$.

\section{Analysis of antisense AtDCL expression}

To further analyze AtDCL function, we generated transgenic Arabidopsis plants that constitutively expressed antisense AtDCL transcript. When selected on Murashige and Skoog medium containing hygromycin, true transformants with healthy roots segregated into normal green and albino seedlings. The latter grew

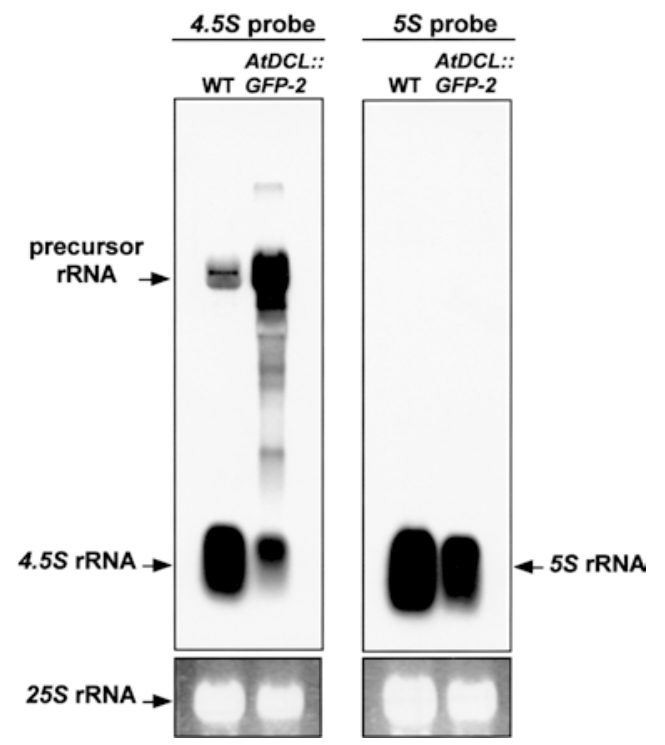

Fig. 5 Effect of overexpression of AtDCL : GFP on chloroplast gene expression in $A$. thaliana. Northern analysis of $4.5 S$ and $5 S$ rRNA expression. Total RNA samples from wild-type $(W T)$ and AtDCL ::GFP-2 mutant (AtDCL::GFP-2) leaves were fractionated on agarose gels, transferred to nylon membrane and hybridized with labeled probes for $4.5 S$ and $5 S$ rRNA. After ethidium bromide coloration of the gel, the cytosolic $25 S$ rRNA is shown in the lower panels as a loading control

slowly and died before true leaves were formed which suggests that expression of antisense AtDCL is lethal.

The survivor T1 transformants were transferred to soil. From $26 \mathrm{~T} 1$ independent transgenic lines, 12 became chlorotic after 2 weeks and were further analyzed. They gave rise to few seeds and their T2 progeny segregated into normal and chlorotic seedlings characterized by variation in cotyledon number. Fiveweek-old siblings of the T2 progeny of one of the 12 transformants are shown in Fig. 6a (normal plant) and $6 \mathrm{~b}$ (mutant plant). Mutant plants grew extremely slowly, had chlorotic flower buds and died after 7-8 weeks (Fig. 6c). In contrast, the normal progeny showed developmental defects at a more advanced stage: yellow inflorescence (Fig. 6d), initiation of many axillary meristems at the same place (Fig. 6e), shoot apical meristem arrested (Fig. 6f), stem fasciation (Fig. 6g) and fasciated siliques (data not shown). We assume that these plants still express the AtDCL gene albeit at reduced levels, while it is completely off in the mutant progeny exhibiting chlorosis at an early stage. Together, these data show that overexpression of antisense AtDCL causes chlorosis throughout the vegetative cycle of the plant and affects normal plant development.

RNA gel blot analysis was used to investigate the effect of overexpression of antisense $A t D C L$ on the steady-state level of $4.5 \mathrm{~S}$ rRNA. Leaf tissue was collected for total RNA extraction from wild-type and antisense AtDCL mutant plants. Figure 7a shows that $4.5 S$ rRNA accumulation was dramatically reduced in antisense $A t D C L$ mutant plants in comparison with the 
Fig. 6a-g T2 progeny of the antisense $A t D C L-11$ line of A.thaliana harboring the antisense $A t D C L$ transgene. a Five-week-old normal plant. b Five-week-old mutant plant. cEight-week-old mutant plant. d Six-week-old normal plant with yellow inflorescence (left) compared to wild-type plant (right).e Eight-week-old normal plant with many axillary meristems initiated at the same place (arrows).f Eight-week-old normal plant with arrested shoot apical meristem (arrowhead). g Eight-week-old normal plant with stem fasciation (arrow)
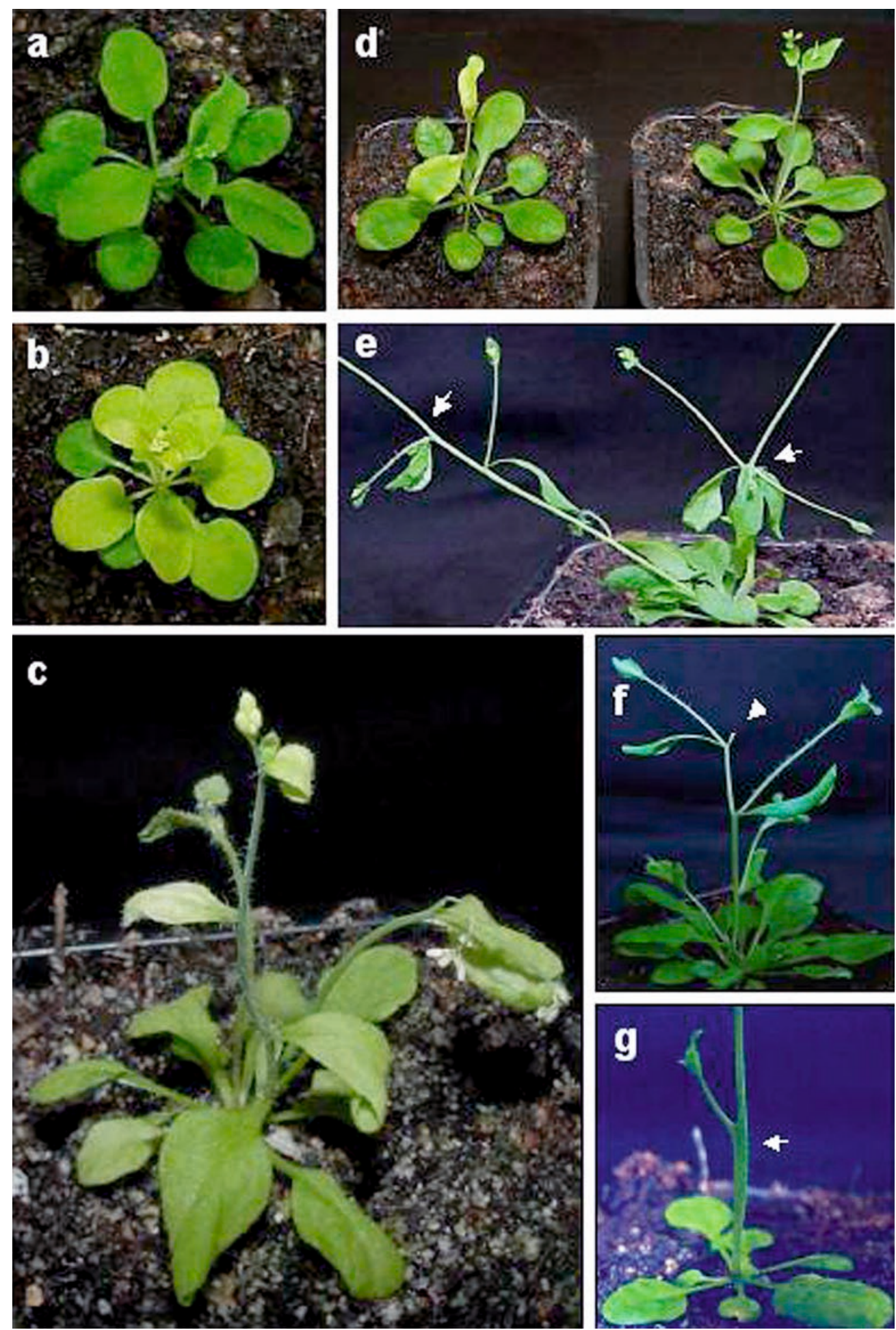

wild type, which is consistent with previous data from overexpression of the AtDCL::GFP fusion.

We then tested the effect of the overexpression of antisense AtDCL on the accumulation of plastid proteins. Total leaf protein was extracted from mutant plants of two independent lines (antisense AtDCL-11 and $A t D C L-21$ ), and protein gel blot analysis was performed using antibodies raised against plastid proteins.
While POR protein accumulated to near normal level, a strong reduction was observed in the abundance of CF $1 \alpha$, PSI-D and LHCII proteins (Fig. 7b). This is consistent with the effect of $d c l-m$ mutation on the accumulation of plastid proteins in tomato (Bellaoui et al. 2003).

Together, these results demonstrate that overexpression of antisense $A t D C L$ affects $4.5 S$ rRNA processing 
a

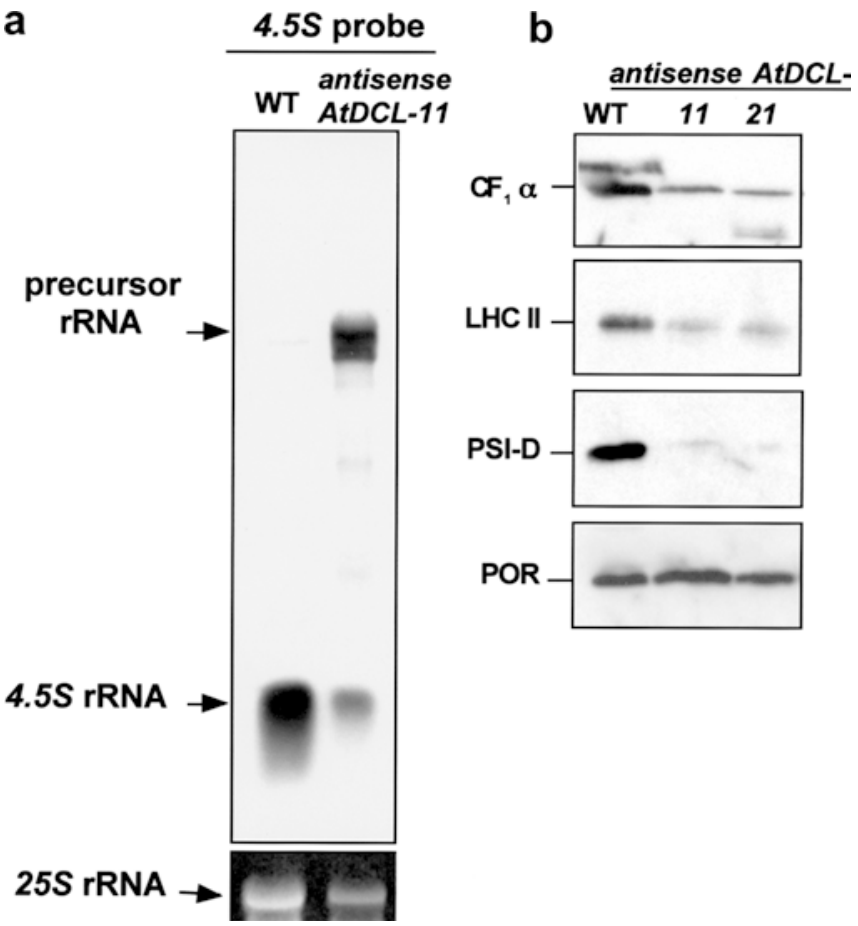

Fig. 7a, b Effect of overexpression of antisense AtDCL on chloroplast gene expression. a Total RNA was prepared from wild-type $(W T)$ and antisense AtDCL mutant (line 11) leaves and analyzed by northern blot hybridization for the expression of $4.5 \mathrm{~S}$ rRNA. After ethidium bromide coloration of the gel, the cytosolic $25 \mathrm{~S}$ rRNA is shown in the lower panel as a loading control. b Immunoblot analysis of plastid proteins. Equal amounts of total protein from wild-type $(W T)$ and antisense AtDCL mutant (lines 11 and 21) plants were separated on 12 polyacrylamideSDS gels and immunoblotted with antisera raised against the $\alpha$-subunit of ATP synthase $(C F 1 \alpha)$, the light-harvesting chlorophyll $a / b$-binding protein of photosystem II $(L H C I I)$, the D subunit of photosystem I (PSI-D), and protochlorophyllide oxidoreductase $(P O R)$

and strongly suggest that AtDCL protein is involved in plastid rRNA maturation.

\section{Discussion}

Five sequences with homology to tomato DCL have been identified in the Arabidopsis genome. A very high degree of conservation was seen at the DCL-domaincontaining C-terminus of these sequences. However, no significant amino acid sequence similarity between these proteins was found at the $\mathrm{N}$ terminus, which suggests that the Arabidopsis homologs of DCL do not have identical activities. We assume that the different DCLlike proteins are functionally distinct members of a gene family sharing a common domain. Among those sequences, AtDCL exhibited the highest amino acid sequence similarity throughout its length with tomato DCL. We have demonstrated that, like tomato DCL, AtDCL is localized in the plastid. Expression analysis showed that $D C L$ and $A t D C L$ are normally expressed not only in photosynthetic but also in non-photosyn- thetic tissue, suggesting that these genes are required for the development of different plastid types in different organs. Our findings that AtDCL and tomato DCL have the same pattern of expression and that are both localized in the same organelle suggest that they perform the same function.

To clarify the in vivo function of the AtDCL gene we investigated the physiological consequence of overexpressing AtDCL antisense RNA and AtDCL::GFP fusion in transgenic Arabidopsis plants. In both strategies, the deregulation of $A t D C L$ gene expression caused multiple phenotypes. Chlorosis is the main developmental defect phenotype observed in transgenic plants. The chlorosis is the result of reduced chlorophylls and carotenoids, which reflect a block of chloroplast development. Deregulation of AtDCL gene expression caused other phenotypes such as sterile flowers, abnormal cotyledon development, fasciated siliques, arrested shoot apical meristem, and stem fasciation. This defect in chloroplast biogenesis that affects plant organ development is similar to that observed in many chloroplast developmental mutants. For instance, null mutations of the nuclear-encoded chloroplast genes EDD1, SLP, Emb506 and DCL cause embryo abortion (Uwer et al. 1998; Albert et al. 1999; Apuya et al. 2001; Despres et al. 2001; Bellaoui et al. 2003). Similarly, mutations that block chloroplast biogenesis and alter palisade cell morphogenesis have been identified, and suggest that chloroplast development also regulates leaf differentiation (Reiter et al. 1994; Chatterjee et al. 1996; Babiychuk et al. 1997; Keddie et al. 1996; Wang et al. 2000). Because the primary defect in all of these mutants appears to be in chloroplast development, we suggest that these genes are involved in the initial events of plastid biogenesis, and that essential compounds for organ development are produced by the early-developed plastid.

We have previously shown that a defect in plastid rRNA processing is associated with $d c l-m$ mutation, and therefore we suggested that the alteration of rRNA maturation is the primary lesion responsible for the blockage of chloroplast biogenesis in the $d c l-m$ mutant (Bellaoui et al. 2003). In this paper we have shown that overexpressing the antisense RNA of the AtDCL gene affected rRNA processing. The same defect was observed when AtDCL::GFP fusion protein was overexpressed in transgenic Arabidopsis plants. Our data suggest that AtDCL activity might be required for rRNA maturation and provide further support for the proposed role of tomato $D C L$ gene in rRNA processing (Bellaoui et al. 2003). In Chlamydomonas, a mutant defective in chloroplast $23 S \mathrm{rRNA}$ maturation has been reported (Boynton et al. 1970; Herrin et al. 1990). Holloway et al. (1998) have shown that the primary defect in this mutant, termed ac20, is associated with the maturation of the 23S rRNA itself.

A defect in chloroplast rRNA processing was also observed in other mutants such as $h c f 7$ in maize and dall2 in Arabidopsis (Barkan 1993; Bisanz et al. 2003). In the $h c f 7$ mutant, maturation of the $16 \mathrm{~S}$ rRNA is blocked and 
the $16 S$ RNA precursor accumulated in the monosome fraction of a polysome gradient, thereby reducing chloroplast translation efficiency (Barkan 1993). In dall-2, a defect in the maturation of $16 S$ and $23 S$ rRNAs was observed, suggesting that Dall protein is involved in plastid rRNA processing (Bisanz et al. 2003). While chloroplast mRNA processing has been extensively investigated, relatively little is known about plastid rRNA maturation. To our knowledge, $h c f 7, a c 20$, dall-2 and $d c l-m$ are the only examples of mutants that are defective in chloroplast rRNA processing, and are ideal tools for determining the enzymes that are involved in this process. Like tomato DCL, AtDCL does not contain obvious RNA-binding domains and does not show similarity to any known nuclease. Preliminary two-hybrid screening experiments show that AtDCL protein interacts with proteins known to be involved in RNA metabolism and ribosome biogenesis. When these interactions are confirmed, genetic and molecular analyses of the candidate enzymes should be helpful for dissecting the molecular mechanism of rRNA processing.

Acknowledgments We thank Chantal Ebel, Arnaud Galichet, Felix Kessler and Grant W. Brown for stimulating discussions and critical reading of the manuscript. We also thank Katalin Konya and Sabine Klarer for maintaining the plants. This work was supported in part by an HFSP long-term fellowship to M.B. and by funds from the Swiss Federal Institute of Technology of Zurich to W.G.

\section{References}

Albert S, Despres B, Guilleminot J, Bechtold N, Pelletier G, Delseny M, Devic M (1999) The EMB506 gene encodes a novel ankyrin repeat containing protein that is essential for the normal development of Arabidopsis embryos. Plant J 17:169-179

Apuya NR, Yadegari R, Fischer RL, Harada JJ, Zimmerman JL, Goldberg RB (2001) The Arabidopsis embryo mutant schlepperless has a defect in the chaperonin-60alpha gene. Plant Physiol 126:717-730

Ausubel M, Brent R, Kingston RE, Moore DD, Seidman JG, Smith JA, Struhl K (1990) Current protocols in molecular biology. Wiley, New York

Babiychuk E, Fuangthong M, Van Montagu M, Inze D, Kushnir S (1997) Efficient gene tagging in Arabidopsis thaliana using a gene trap approach. Proc Natl Acad Sci USA 94:12722-12727

Barkan A (1993) Nuclear mutants of maize with defects in chloroplast polysome assembly have altered chloroplast RNA metabolism. Plant Cell 5:389-402

Barkan A (1998) Approaches to investigating nuclear genes that function in chloroplast biogenesis in land plants. Methods Enzymol 297:38-57

Bellaoui M, Keddie JS, Gruissem W (2003) DCL is a plant-specific protein required for plastid ribosomal RNA processing and embryo development. Plant Mol Biol 53:531-543

Bisanz C, Begot L, Carol P, Perez P, Bligny M, Pesey H, Gallois JL, Lerbs-Mache S, Mache R (2003) The Arabidopsis nuclear $D A L$ gene encodes a chloroplast protein which is required for the maturation of the plastid ribosomal RNAs and is essential for chloroplast differentiation. Plant Mol Biol 51:651-663

Bonhomme S, Budar F, Férault, Pelletier G (1991) A Nco I fragment of Ogura radish mitochondrial DNA is correlated with cytoplasmic male-sterility in Brassica cybrids. Curr Genet 19:121-127
Boynton JE, Gillham NW, Burkholder B (1970) Mutations altering chloroplast ribosome phenotype in Chlamydomonas. II. Non-mendelian mutations. Proc Natl Acad Sci USA 67:1505-1512

Chatterjee M, Sparvoli S, Edmunds C, Garosi P, Findlay K, Martin C (1996) DAG, a gene required for chloroplast differentiation and palisade development in Antirrhinum majus. EMBO J 15:4194-4207

Clough SJ, Bent AF (1998) Floral dip: a simplified method for Agrobacterium-mediated transformation of Arabidopsis thaliana. Plant J 16:735-743

Despres B, Delseny M, Devic M (2001) Partial complementation of embryo defective mutations: a general strategy to elucidate gene function. Plant J 27:149-159

Edwards K, Bedbrook J, Dyer T, Kössel H (1981) 4.5S rRNA from Zea mays chloroplasts shows structural homology with the $3^{\prime}$ end of prokaryotic 23S rRNA. Biochem Int 2:533-538

Grelon M, Budar F, Bonhomme S, Pelletier G (1994) Ogura cytoplasmic male-sterility (CMS)-associated orf138 is translated into a mitochondrial membrane polypeptide in malesterile Brassica cybrids. Mol Gen Genet 243:540-547

Harris EH, Boynton JE, Gillham NW (1994) Chloroplast ribosomes and protein synthesis. Microbiol Rev 58:700-754

Herrin DL, Chen YF, Schmidt GW (1990) RNA splicing in Chlamydomonas chloroplasts. Self-splicing of $23 S$ preRNA. J Biol Chem 265:21134-21140

Holloway SPa, Herrin DL (1998) Processing of a composite large subunit rRNA: studies with Chlamydomonas mutants deficient in maturation of the 23S-like rRNA. Plant Cell 10:1193-1206

Keddie JS, Carroll B, Jones JD, Gruissem W (1996) The DCL gene of tomato is required for chloroplast development and palisade cell morphogenesis in leaves. EMBO J 15:4208-4217

Kössel H, Edwards K, Koch W, Langridge P, Schiefermayr E, Schwarz Z, Strittmatter G, Zenke G (1982) Structural and functional analysis of an rRNA operon and its flanking tRNA genes from Zea mays chloroplasts. Nucleic Acids Res Symp Ser 11:117-120

Kössel H, Natt E, Strittmatter G, Fritzsche E, Gozdzicka-Jozefiak A, Przybyl D (1985) Structure and expression of rRNA operons from plastids of higher plants. In: van Vloten-Doting L, Groot GSP, Hall TC (eds) Molecular form and function of the plant genome. Plenum, New York, pp 183-198

Laemmli UK (1970) Cleavage of structural proteins during the assembly of the head of bacteriophage T4. Nature 227:680-685

Reiter RS, Coomber SA, Bourett TM, Bartley GE, Scolnik PA (1994) Control of leaf and chloroplast development by the Arabidopsis gene pale cress. Plant Cell 6:1253-1264

Rochaix JD, Darlix JL (1982) Composite structure of the chloroplast $23 S$ rRNA genes of Chlamydomonas reinhardii. J Mol Biol 159:383-395

Rodriguez-Concepcion M, Yalovsky S, Zik M, Fromm H, Gruissem W (1999) The prenylation status of a novel plant calmodulin directs plasma membrane or nuclear localization of the protein. EMBO J 18:1996-2007

Spangenberg G, Potrykus I (1995) Polyethylene glycol-mediated direct gene transfer to tobacco protoplasts and regeneration of transgenic plants. In: Potrykus I, Spangenberg G (eds) Gene transfer to plants. Springer, Berlin Heidelberg New York

Strittmatter G, Kössel H (1984) Cotranscription and processing of $23 S, 4.5 S$ and $5 S$ rRNA in chloroplasts from Zea mays. Nucleic Acids Res 12:7633-7647

Uwer U, Willmitzer L, Altmann T (1998) Inactivation of a glycyltRNA synthetase leads to an arrest in plant embryo development. Plant Cell 10:1277-1294

Wang Y, Duby G, Purnelle B, Boutry M (2000) Tobacco VDL gene encodes a plastid DEAD box RNA helicase and is involved in chloroplast differentiation and plant morphogenesis. Plant Cell 12:2129-2142 